\title{
Design of Load-sensitive Multi-way Valve Test System
}

$$
\text { Liping } \mathrm{Xu}^{1, \mathrm{a}} \text {, Liujin } \mathrm{Cai}^{1, \mathrm{~b},{ }^{*}} \text { and Xiaoqing } \mathrm{Nan}^{1, \mathrm{c}}
$$

${ }^{1}$ Henan University of Science and Technology, School of Mechatronics Engineering, Henan, Luoyang, 471000, China

axxpzz@163.com, byycailj@163.com, ‘nxq_chn@163.com

${ }^{*}$ Corresponding author

Keywords: Load-sensitive; Multi-way Valve; Test; STM32F103; LabVIEW

\begin{abstract}
As the load-sensitive multi-way valve is being used more widely, its performance is becoming more and more important in the hydraulic system. Thus it is urgent to design a low-cost system which can quickly measure the performance of load-sensitive multi-way valve. Taking the load-sensitive multi-way valve as the test target, a hydraulic test circuit was designed to complete the functional experiment. The STM32 MCU was used as the core of hardware circuit, and the high-performance W5500 Ethernet chip as a communication module. The control software was developed by LabVIEW platform, on which the experimenters retrieve, observe, store and print the online testing data of the valve. A qualified load-sensitive multi-way valve specializing in agricultural machinery was tested to verify the designed system, the result shows that the designed system is stable and reliable.
\end{abstract}

\section{Introduction}

With the rapid development of engineering machinery, load sensitive phenomenon has been brought in the engineering machinery dynamical system. It is widely used in engineering machinery due to its high energy efficiency. Nowadays, domestic scholars have designed a lot of multi-way valve systems, but the hydraulic test system based on the trait of load-sensitive multi-way valve and the accomplishment of the relevant performance tests is rarely reported. For this reason, it is necessary to realize the traits of a load-sensitive multi-way valve which is applicable for agricultural machinery operation and invent a set of testing system for experimenting on the load-sensitive multi-way valve.

The testing system with software developed by LabVIEW is always adopts with the matched data acquisition card of NI company. Although the data acquisition card is easily to be programmed, its cost is high. In this article, single chip microcomputer STM32 is used as the control center of the testing system hardware, LabVIEW is used as the software design platform, and the data transmission medium is W5500 embedded Ethernet controller. Due to its low cost, short development cycle, users can achieve the Ethernet data communication through W5500 just by using a simple Socket program instead of operating a complicated Ethernet controller. It is low-cost , short development cycle, easily-integrated and applicable for remote transmission. The test result shows that the testing system can experiment on the performance index of load-sensitive multi-way valve effectively ${ }^{[1][2]}$.

\section{The Design of Load-sensitive Multi-way Valve Hydraulic System}

Brief Introduction of the Tested Valve. Load-sensitive multi-way valve is a small hydraulic system combined with various kinds of valves. It is always composed of oil inlet connection, working connection and terminal connection. The number of working connection is customized, and it is determined by the needed external load as the engineering machinery real working ${ }^{[3]}$. Shown in picture 1, No.15 is a load-sensitive multi-way valve used on an agricultural machinery with one working connection. Its oil inlet connection includes of manual directional valve, shuttle valve and pressure compensation valve. The pressure compensation valve combines with the instant sectional area of the pilot valve in the directional control valve to regulate the flow. The 
load-sensitive multi-way valve is tested in a good performance, its maximum flow $\mathrm{q}_{\max }=80 \mathrm{~L} / \mathrm{min}$, the diameter of the oil port $\mathrm{A}$ and $\mathrm{B}$ is $22 \mathrm{~mm}, \mathrm{P}=1.5 \sim 17.5 \mathrm{MPa}$.

The Design of Hydraulic Testing Loop. During the design of load-sensitive multi-way valve testing system, we considered the tested valve's self-load-sensitive feature and the essence of multi-way. According to the B/T8729-2013 hydraulic multi-way directional valve machinery industrial standard and relevant industrial standards, the test theory figure shown in Fig.1 was designed. Proportional overflow valve is used to set up the pressure of the whole system in order to reach the testing requirement ${ }^{[4][5]}$. Nevertheless the pressure sensor and the flow sensor can choose different measuring range according to the actual situation. In view of the smaller flow of the load-sensitive port $\mathrm{L}_{\mathrm{S}}$, a flow sensor is chosen, which has a higher precision. For this reason, ZHM $01 / 21)^{*}$,with the measuring range of $0.02-3 \mathrm{~L} / \mathrm{min}$ and the measurement accuracy of $\pm 0.5 \%$, produced by Shanghai Kem observation and control technology limited company is chosen to use. The testing system is designed to reach all kinds of valve performance tests, besides conventional detection such as safety valve performance, pressure loss, disruptive test, back pressure test, communicative performance, internal leakage, load sensing performance, steady state test, transient test, operating force and so on, the flow change of fretting behavior test is achieved additional.

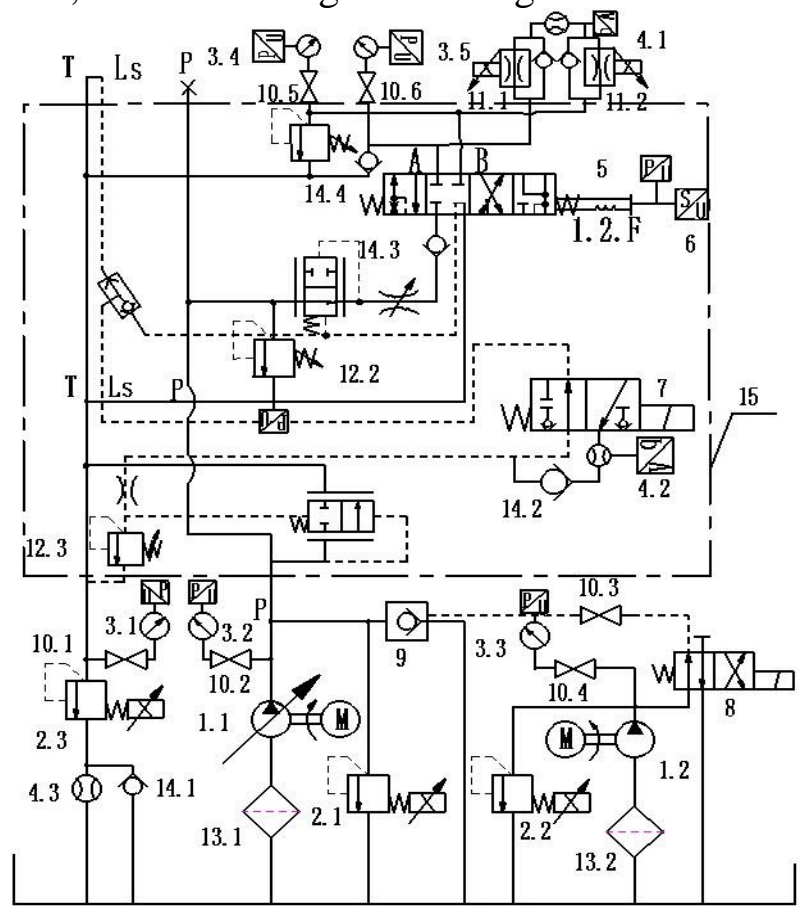

Figure.1 Hydraulic testing circuit

1.1 1.2 fluid pump; 2.1 2.3 proportional overflow valve; $3.1 \sim 3.5$ pressure gage and pressure sensor;

4.1 4.3 flow gage and flow sensor; 5 tension \& compression sensor; 6 displacement sensor;

7 electromagnetic ball valve; 8 electromagnetic valve; 9 discontinuity loading valve;

10.1 10.6 stop valve; 11.1 11.2 unidirectional proportional throttle valve;

12.1 12.3 overflow valve; 13.1 13.2 filter; 14.1 14.4 unidirectional valve;

15 tested load-sensitive multi-way valve

Brief Introduction of a Main Test Program. A main program testing method according to the hydraulic testing loop is introduced as follows, shown in Fig.1.

Fretting behavior test: adjusting safety valve of the tested valve to nominal pressure and turn off all overload valves. (1) Pressure fretting behavior from $\mathrm{P}$ to $\mathrm{T}$ : plugging the tested valve oil port $\mathrm{B}$ and $\mathrm{A}$, letting nominal flow rate going through port $\mathrm{P}$, the slide valves move from the neutral position to every commutation position slowly. At this time the relevant pressure parameter varying with the movement of oil port $\mathrm{P}$ is measured. (2) Flow fretting behavior from $\mathrm{P}$ to $\mathrm{A}(\mathrm{B})$ : let oil port $\mathrm{P}$ go through neutral flow, while valves move from the neutral position to every commutation 
position slowly. Keeping the load of overflow valve12.1 is $75 \%$ of the neutral pressure at the same time, the sensor measures the relevant flow parameter during the movement. (3)Flow fretting behavior from $\mathrm{A}(\mathrm{B})$ to $\mathrm{P}$ : let port $\mathrm{A}(\mathrm{B})$ feed with oil and go through neutral flow, set up proportional overflow valve 2.1 , turn the system pressure to the $75 \%$ of the neutral pressure. Slide valves move from the neutral position to every commutation position slowly. And then measure the relevant flow during the movement.

\section{Design of the Testing Hardware Part}

The test system is controlled by upper computer and lower computer. The system operation is shown in Figure 2. Signals of the tested system such as flux, displacement, pressure, etc. are measured by relevant sensors. Sensors' data go into STM32F103 after being voltage-sampled and converted by signal conditioning circuit and A/D conversion module. Afterwards the digital signals converted will be computed by specific control algorithm in the software. The result outputs to the W5500 net card module, which brings with external TCP/IP protocol, to achieve the data transmission to industrial personal computer (IPC). At the same time, the overflow valve can be adjusted by PWM signal, and the result will be sent to IPC. When the testing system pressure or flow changes, IPC with LabVIEW software will send new signals to W5500 and then delivered to STM32F103, and to realize the signal control and feedback consequently ${ }^{[6]}$.

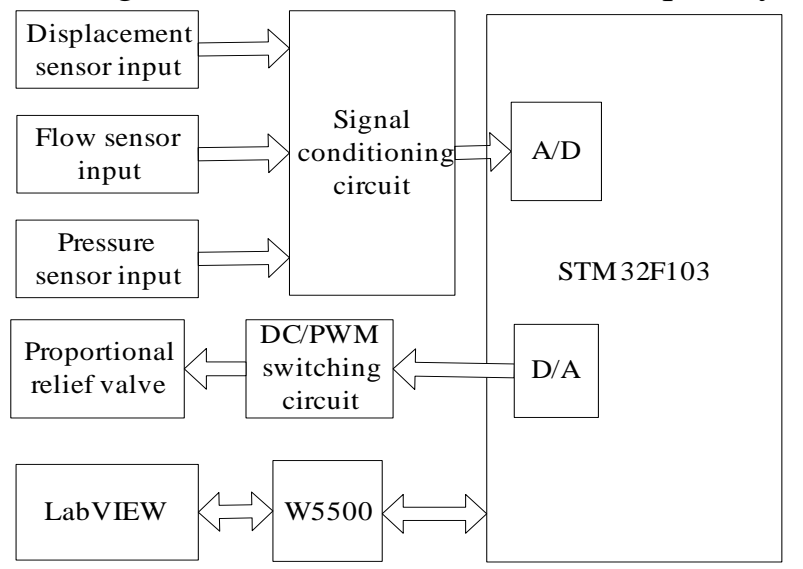

Figure.2 Block diagram of testing system principle

The hardware of lower computer mainly consists of controller, signal conditioning circuits and control program. The micro-controller STM32F103 in the series of STM32 is chosen as the controller which is low power-loss and reasonable price. The ADC of STM32 is a 12-bit successive approximation analog-to-digital converter. Its measuring precision is high of $\pm 0.25 \%$, which is less than the precision of the chosen sensor and meet the Testing Grade A of test bed. Its testing result is more accurate. The control program, using $\mathrm{C}$ programming language, is designed with Keil Vision5 platform. Its signal conditioning circuits include partial pressure, capacitor filtering and guarding circuit. Due to the module converting voltage of the controller STM32F103 is 0-3.6V, while the output signal current of sensors are all 4-20mA, therefore the conversion of each sensor's output current of the partial pressure in the signal conditioning circuit is shown in Fig.3.

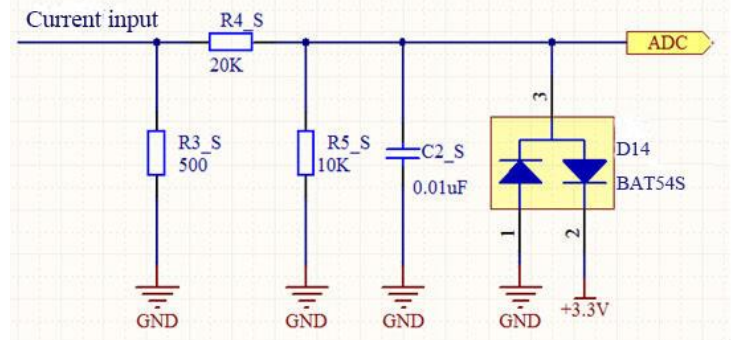

Figure.3 Current input signal conditioning circuit 


\section{Design of the Testing Software Part}

The higher computer software of the testing system is written by LabVIEW. In consider of the limitation of laboratory testing condition, the testing projects mainly include safety valve transient response test, steady state test, fretting behavior test, internal leakage and back pressure test. Steady state test, for instance, as is shown in Figure 4, is the operation interface of steady state test.

All of the operation interface meets with the functions mainly about: (1) store the essential information such as the name of operating personnel and time. (2) complete the transient response test, steady state test and operating force test of load-sensitive multi-way valve.(3) users operate in real time and send commands for lower computer to control the proportional overflow valve.(4) collect data and realize the displaying, saving and printing out in LabVIEW interface, as shown in Fig.4. The program is shown in figure 5.

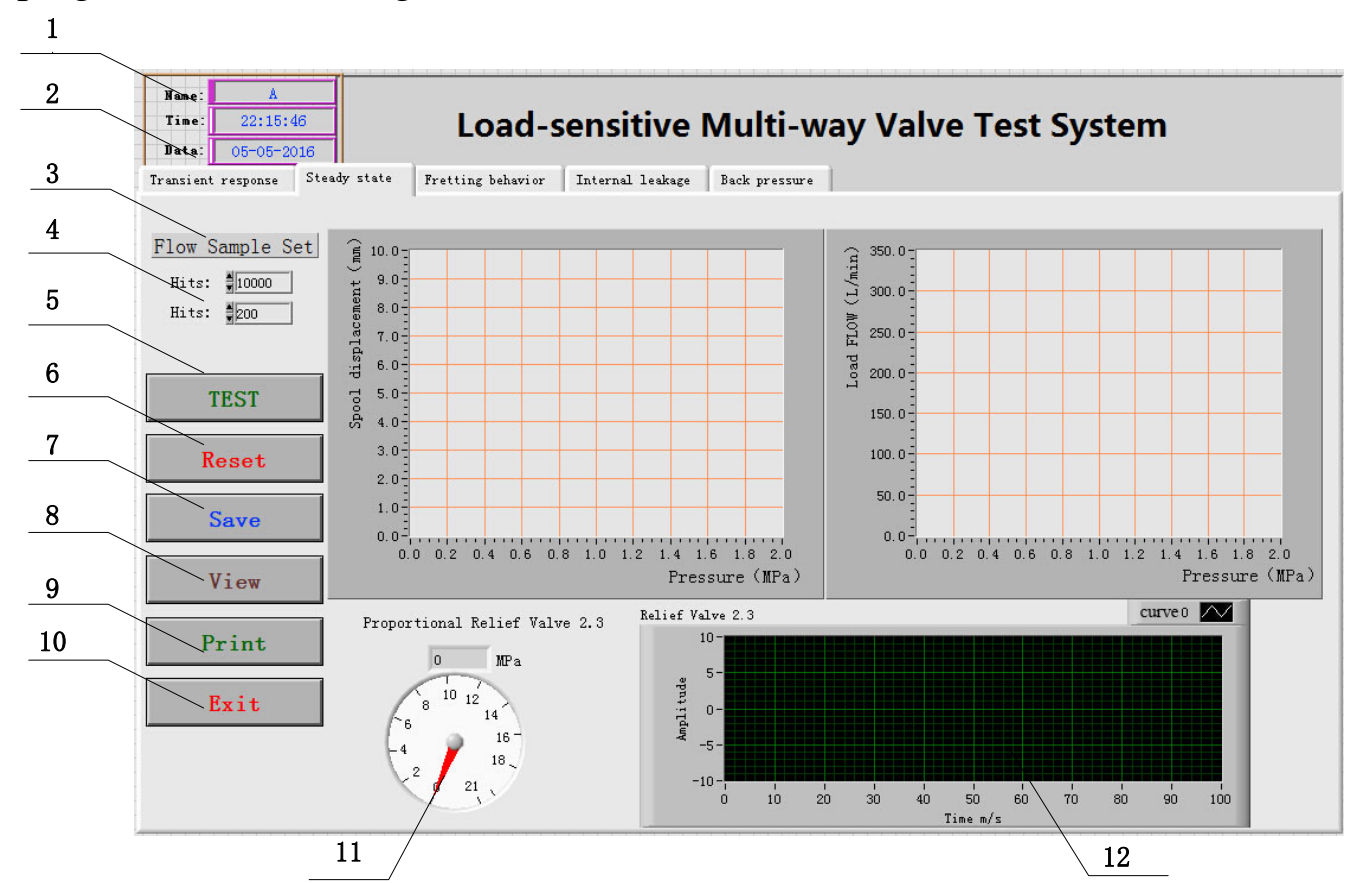

Figure. 4 The front panel of Load sensitive multi-way valve steady-state performance test 1 The experimenter information; 2 Test project; 3 Hydraulic control pressure\&valve core displacement; 4 Sample set; 5 Start; 6 Reset; 7 Save; 8 View; 9 Print; 10 Exit;

11 Pressure gauge; 12 Pressure and time response curves

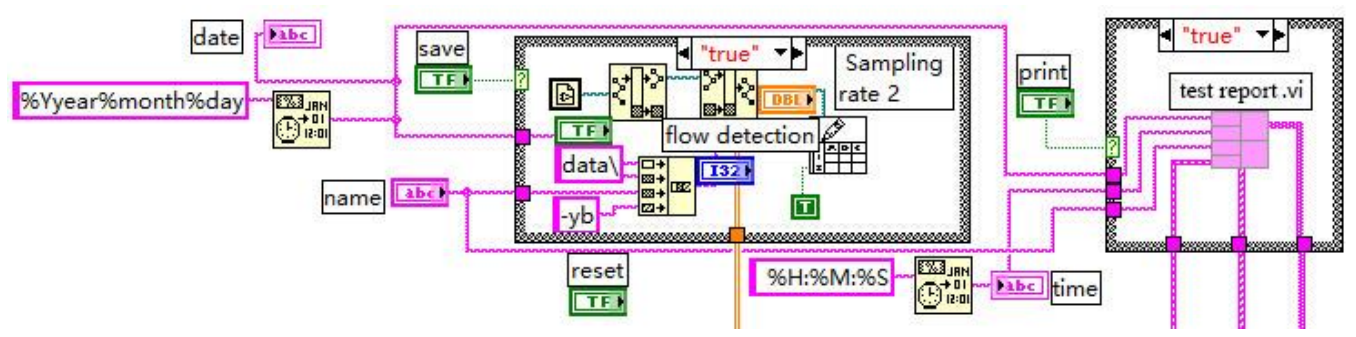

Figure.5 Save, print and time display program

\section{Safety Valve Transient Response Test}

Since testing projects are too many, this article just introduces the safety valve transient response test and its test result of load-sensitive multi-way valve. The specific methods and procedures of the experiment: first, turn off overflow valve2.1, plug oil port A and B of the tested valve. And then put slide valve at commutation position, adjust the safety valve to $16 \mathrm{MPa}$, turn on pump 1.2 and adjust proportional overflow valve 2.2 and control the pressure to speed up the operation of discontinuity loading valve. Next, open all of the stop valve 10.3, put electromagnetic valve 8 at the original position to reduce the inlet pressure of the tested valve to the initial pressure, and then move 
electromagnetic valve to the right rapidly. At last, turn off discontinuity loading valve promptly, and produce a pressure gradient at the inlet of the tested valve to meet the transient condition. Table 1 is the standard safety valve index, the index of the tested valve should keep within the range of Table 1.The measured result is shown in Fig 6.The value of the crest is $18.9 \mathrm{MPa}$ and the actual measured pressure is $15.7 \mathrm{MPa}$. Detailed experimental results are as follows:

$$
\begin{aligned}
& \text { pressure oscillation: } \Delta \mathrm{P}= \pm 0.4 \mathrm{MPa}< \pm 0.5 \mathrm{MPa} \quad \text { (1) } \\
& \text { pressure overshooting rate: } \delta=\frac{18.9 \mathrm{MPa}-15.7 \mathrm{MPa}}{15.7 \mathrm{MPa}} \times 100 \%=20.38 \%<25 \% \\
& \text { transient recovery time: } \mathrm{t}=0.16 \mathrm{~s}<0.2 \mathrm{~s}
\end{aligned}
$$

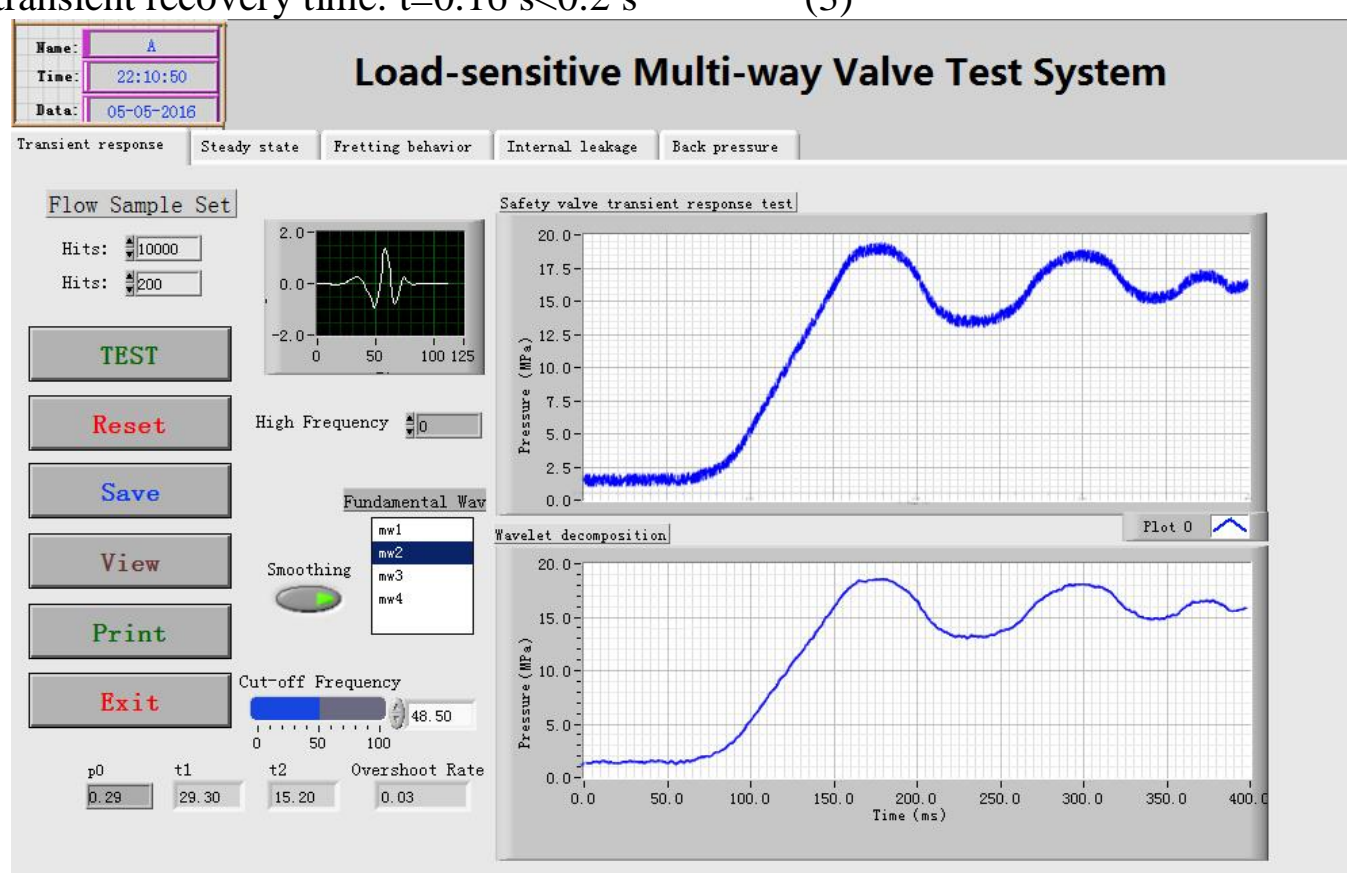

Figure.6 The relief valve transient response experiment operation interface

The result of safety valve transient response test shows the testing result is up to standard and thus the reliability of testing system is high.

Table 1 Standard safety valve index

\begin{tabular}{lcccc}
\hline $\begin{array}{l}\text { Safety valve } \\
\text { performance }\end{array}$ & \multicolumn{4}{c}{ Nominal pressure (MPa) } \\
\cline { 2 - 5 } & 16 & 20 & 25 & 31.5 \\
\hline $\begin{array}{l}\text { Pressure oscillation } \\
\text { (MPa) }\end{array}$ & \pm 0.5 & \pm 0.6 & \pm 0.7 & \pm 0.8 \\
$\begin{array}{l}\text { Pressure overshooting } \\
\text { rate (\%) }\end{array}$ & & & $\leqq 25$ & \\
$\begin{array}{l}\text { Transient recovery } \\
\text { time(s) }\end{array}$ & $\leqq 0.2$ & $\leqq 0.22$ & $\leqq 0.24$ & $\leqq 0.25$ \\
\hline
\end{tabular}

\section{Conclusion}

The article introduces a special load-sensitive multi-way valve for an agricultural machinery, designs the diagram of the load-sensitive multi-way valve principle, writes the testing software based on LabVIEW and the hardware testing system with STM32 and W5500 as the core. It can not only shorten the development period of the system and save the cost. After that, it introduces the safety valve transient response test of the tested valve and gets the experiment data to compare with the standard data. The final testing result shows that this testing system is reliable and provides a reference for the research of load-sensitive multi-way valve testing system. 


\section{Acknowledgements}

The authors gratefully acknowledge natural science foundation of henan university of science and technology for financial support of this research work. (2015QN017)

\section{References}

[1] Zhang Yong, Dong Haobin .Design of a seismic data acquisition system based on STM32 and LabVIEW[J].Measurement Control Technology and Instruments, 2012,38 (10):73-75 (In Chinese).

[2] Kim S D, Cho H S.A suboptimal controller design method for the energy efficiency of a load-sensing hydraulic servo system[J]. Journal of dynamic systems, measurement, and control, 1991.113(3):487-493.

[3].Zeng Hong, Dang Panpan. Design of Embedded Ethernet Interfaces Based on W5500[J]. Network Security Technology and Application. 2015 (2):36-37(In Chinese).

[4] Hydraulic fluid power-Multiple directional valve[S].Beijing: China Machine Press, 2013(In Chinese).

[5] Zhang Jun. Common Failures Analysis of Load-sensitive Proportional Multi-way Valve [J]. Chinese Hydraulics and Pneumatics. 2015 (In Chinese).

[6] Wu D, Burton R, Schoenau G, et al. Establishing operating points for a linearized model of a load sensing system [J]. International Journal of Fluid. 